\title{
A Versatile Protocol for Functionalization of Covalent Organic Frameworks via Suzuki-Miyaura Cross-Coupling
}

Qiaobo $\mathrm{Liao}^{+}$, Can $\mathrm{Ke}^{+}$, Xin Huang+, Dongni Wang, Qingwen Han, Yifan Zhang, Yiying Zhang, and $\mathrm{Kai} \mathrm{Xi}^{*}$

$\left[{ }^{*}\right]$ Q. Liao, ${ }^{[+]}$C. Ke, ${ }^{[+]}$Dr. X. Huang, ${ }^{[+]}$D. Wang, Q. Han, Y. Zhang, Y. Zhang, Prof. K. Xi,

School of Chemistry and Chemical Engineering

Nanjing University

Nanjing, Jiangsu 210023, P. R. China

E-mail: xikai@nju.edu.cn

$\left[{ }^{+}\right]$These authors contributed equally to this work.

Supporting information for this article is given via a link at the end of the document.

\begin{abstract}
Post-synthetic modification (PSM) is a prevalent and powerful strategy to introduce desired functionalities into covalent organic frameworks (COFs) for functional products, expediting their applications vastly. Herein, we demonstrate a PSM strategy for functionalizing brominated COFs via the well-developed Suzuki-Miyaura crosscoupling. By this protocol, a variety of functionalities were installed into COFs efficiently, while the crystallinity and porosity of $\mathrm{COFs}$ retained well. As a proof-of-concept, BrCOF-2 was modified with trifluoromethyl groups to produce a $\mathrm{SF}_{6}$ adsorbent with remarkably enhanced properties. This facile and versatile approach opens a new door for the synthesis of functional COFs, and greatly expands the scope of their structural design aiming for various properties and applications.
\end{abstract}




\section{Introduction}

Covalent organic frameworks (COFs), a class of crystalline porous polymers, have attracted increasing interest since its initial report in 2005 by Yaghi et al. ${ }^{[1]}$ Constructed by covalently linked organic molecules based on dynamic covalent chemistry and topological design principle, COFs feature low density, high stability, large porosity, ordered, and accessible channels. ${ }^{[2,3]}$ These characteristics endow COFs with potential applications in liquid phase adsorption, ${ }^{[4,5]}$ gas separation, ${ }^{[6,7]}$ energy storage materials, ${ }^{[8,9]}$ electronic devices, ${ }^{[10,11]}$ heterocatalysis ${ }^{[12,13]}$, and drug delivery. ${ }^{[14,15]}$ By introducing functionalities into the defined channels, functional COFs can be obtained aiming at specific applications. Albeit that some functional COFs have been synthesized via the de novo synthesis, this method is not omnipotent for every desired functional COFs due to the inherence uncertainties. ${ }^{[16,17]}$ It is well-known that the solubility, reactivity, and geometry of monomers bearing functional groups are so diversified that tedious trails are necessary for screening different synthetic parameters. ${ }^{[18]}$ To circumvent these obstacles, the post-synthetic modification (PSM) strategy is recently utilized widespread for introducing functional groups onto the pore wall of COFs, paving the way toward various functional COFs. ${ }^{[17,19,20]}$ Fulfilling the stringent criteria of high efficiency and yields, no or inoffensive byproducts, orthogonality, and compatibility, it is not surprising that click chemistry (CC) such as copper-catalyzed azide-alkyne cycloaddition $(\mathrm{CuAAC}),{ }^{[21,22]}$ thiol-ene reactions ${ }^{[23,24]}$ and activated esters ${ }^{[25]}$ are emerging as the mainstream strategies for PSM of COFs. However, the number of commercially available precursors is limited to some extent, thereby restricting the scope of the practical application of CC in the field of COFs modification. ${ }^{[26]}$

Thanks to the seminal and fundamental works of Suzuki ${ }^{[27,28]}$ who have been awarded the Nobel Prize in 2010 , a general, versatile, and highly efficient method for the construction of carbon-carbon (C-C) bond have been wellestablished in recent decades, i.e. Suzuki-Miyaura cross-coupling. Owing to the merits of mild synthetic conditions, high tolerance toward functionalities, ease of handling and separation, stability of precursors as well as orthogonality, ${ }^{[29-31]}$ Suzuki-Miyaura cross-coupling is of board utility in the functionalization of synthetic and natural macromolecules, such as poly (S-vinyl sulfide) derivatives, ${ }^{[32]}$ well-defined polymers, ${ }^{[26]}$ aromatic mainchain polymers, ${ }^{[33]}$ DNA, ${ }^{[34]}$, etc. More intriguingly, an ultra-large library of commercially available reaction partners for Suzuki-Miyaura cross-coupling has been well-established, including thousands of boronic acids and boronate esters, ${ }^{[26]}$ offering much more possibilities for functionalization. Besides, Suzuki monomers can be easily synthesized using many well-developed methods such as Miyaura-Ishiyama borylation. ${ }^{[29]}$ Thus, Suzuki-Miyaura cross-coupling is considered as a promising candidate for functionalizing COFs, which has not yet been explored to the best of our knowledge.

In this article, we report a general and facile strategy for modifying COFs post-synthetically via SuzukiMiyaura cross-coupling (Scheme 1). By exploiting the brominated monomers, two highly crystalline brominated COFs (denoted as BrCOFs) have been obtained with aryl bromine atoms aligned on the 1D channel walls, allowing for the downstream functionalization. Based on Suzuki-Miyaura cross-coupling, a variety of functionalities including methylthio, formyl, carbomethoxy, cyan and trifluoromethyl were successfully introduced into COFs, while the crystallinity and porosity of COFs were well-retained. Furthermore, the BrCOF-2 modified with the trifluoromethyl group showed notably enhanced properties for $\mathrm{SF}_{6}$ adsorption and separation as compared to the pristine BrCOF-2. 


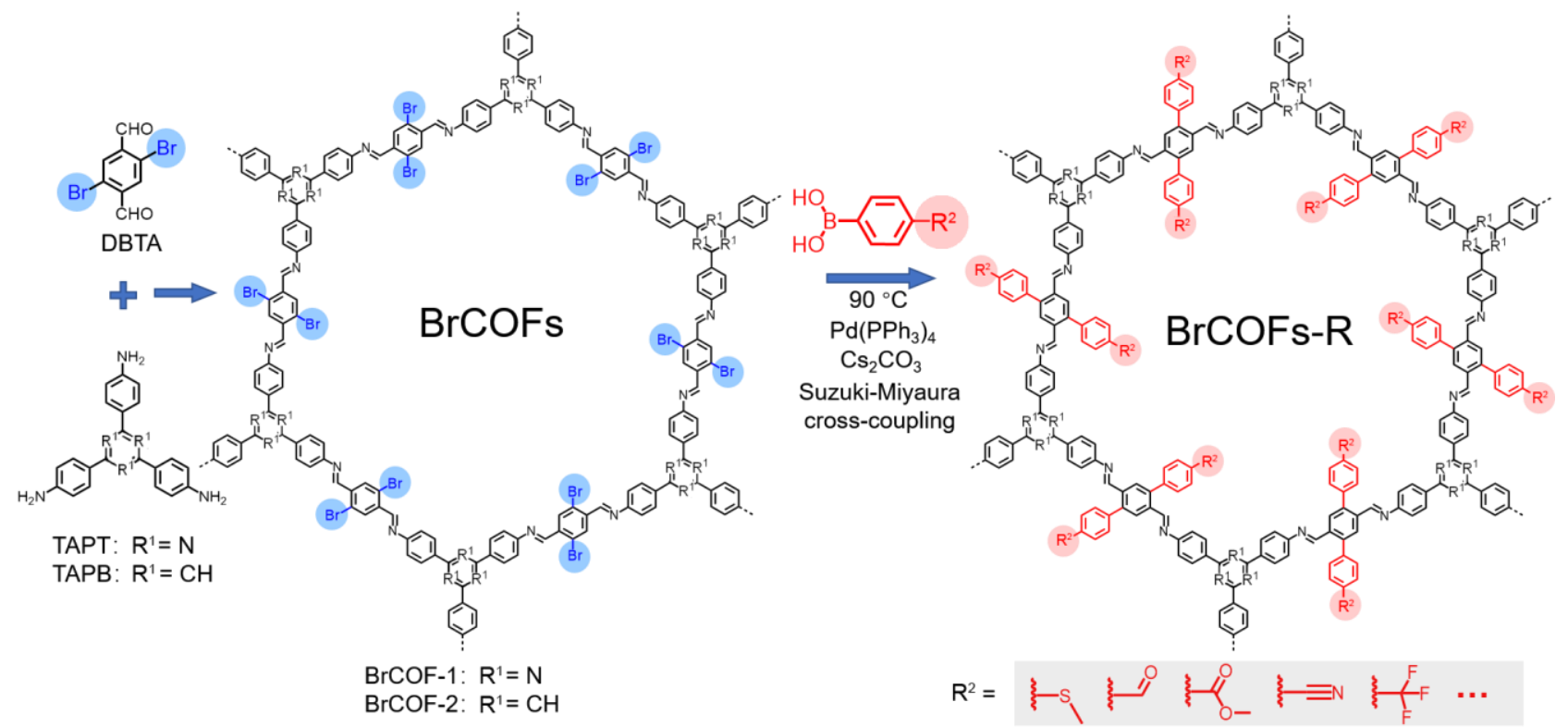

Scheme 1. Schematic representation for the synthesis of BrCOFs and the post-synthetic modification via Suzuki-Miyaura crosscoupling.

\section{Results and discussion}

To verify the concept of functionalization via Suzuki-Miyaura cross-coupling, two prototype brominated COFs denoted as BrCOF-1 and BrCOF-2 were synthesized using the solvothermal condensation method by reactions of 2,5-dibromoterephthaldehyde (DBTA) and 2,4,6-tris(4-aminophenyl)-1,3,5-triazine (TAPT) or 1,3,5-tris(4aminophenyl) benzene (TAPB), respectively (Scheme 1, see Supporting Information-SI for details). According to the Fourier-transform infrared (FT-IR) spectra of the as-synthesized BrCOFs. (Figure S1 and S2), the peaks corresponding to $\mathrm{C}=\mathrm{N}$ stretching vibration at around $1622 \mathrm{~cm}^{-1}$ (for BrCOF-1) and $1618 \mathrm{~cm}^{-1}$ (for BrCOF-2) can be distinguished, confirming the successful construction of imine bonds. Further evidence was provided by solid-state ${ }^{13} \mathrm{C}$ cross-polarization magic-angle-spinning (CP-MAS) NMR spectroscopy of BrCOF-1 and BrCOF-2, in which the signals at around $150 \mathrm{ppm}$ for $\mathrm{C}=\mathrm{N}$ band (154.6 ppm for BrCOF-1 and $151.2 \mathrm{ppm}$ for BrCOF-2) emerged while those for aldehyde group $(\mathrm{C}=\mathrm{O})$ at around $190 \mathrm{ppm}$ disappeared completely (Figure S3 and S4). The scanning electron microscopy (SEM) images show that BrCOF-1 adopts fiber-like morphology while BrCOF-2 is worm-like (Figure S5). These morphologies endow BrCOFs with large outer accessible surfaces and volumes which are beneficial for the infiltration of the modifying molecules. As shown in Figure S6 and S7, both BrCOFs exhibit excellent thermal stability with degradation temperatures up to $416{ }^{\circ} \mathrm{C}$ (BrCOF-1) and $428{ }^{\circ} \mathrm{C}$ (BrCOF-2) determined by thermogravimetric analysis (TGA).

Powder X-ray diffraction (PXRD) patterns of both BrCOFs show strong reflections corresponding to (100) facet at around $2.8^{\circ}\left(2.84^{\circ}\right.$ for BrCOF-1; $2.83^{\circ}$ for BrCOF-2) accompanied by weaker peaks centered at $5.84^{\circ}, 7.57^{\circ}$, $25.4^{\circ}$ (corresponding to (200), (120), (001) facets, respectively) for BrCOF-1 and $4.85^{\circ}, 5.63^{\circ}, 7.48^{\circ}, 24.9^{\circ}$ (corresponding to (110), (200), (120), (001) facets, respectively) for BrCOF-2, verifying the highly crystalline nature of BrCOFs (Figure 1a). The layer distances of BrCOF-1 and BrCOF-2 were calculated to be $0.35 \mathrm{~nm}$ and $0.36 \mathrm{~nm}$, respectively, based on the reflections at $25.4^{\circ}$ and $24.9^{\circ}$ (Figure 1a, insets). In terms of the molecular symmetry of monomers, possible 2D layer structures of BrCOF-1 were constructed based on hcb topology with eclipsed (AA) and staggered (AB) stacking modes using the Materials Studio software package (Figure S8b-c and S9b-c). For both BrCOFs, the simulated PXRD patterns of the AA stacking mode match better with the experimental results compared to those of the $\mathrm{AB}$ stacking mode, proving their actual structures of AA stacking. Furthermore, the Pawley-refinement was applied to define the final unit cells of BrCOFs, yielding good agreement factors $\left(\mathrm{R}_{\mathrm{wp}}\right.$ 
$=5.94 \%$ for BrCOF-1; $\mathrm{R}_{\mathrm{wp}}=6.22 \%$ for BrCOF-2, Figure S8a and S9a). Bromine contents of BrCOF-1 and BrCOF2 determined by energy-dispersive X-ray spectroscopy (EDX) equipped on SEM are $31.49 \pm 5.90 \mathrm{wt} \%$ and 32.39 $\pm 5.84 \mathrm{wt} \%$, respectively, which are in good accordance with calculated values for infinite $2 \mathrm{D}$ structures $(32.11 \mathrm{wt} \%$ for BrCOF-1, $32.60 \mathrm{wt} \%$ for BrCOF-2). Besides, elemental analyses revealed that the nitrogen, carbon and hydrogen contents of these COFs were 11.28\%, 53.18\%, 2.87\% for BrCOF-1 and 5.66\%, 58.76\%, 4.69\% for BrCOF-2, respectively, which are closely similar to theoretical values (BrCOF-1: $11.39 \%$ for $\mathrm{N}, 53.69 \%$ for $\mathrm{C}, 2.46 \%$ for $\mathrm{H}$; BrCOF-2: $5.73 \%$ for $\mathrm{N}, 58.80 \%$ for $\mathrm{C}, 2.88 \%$ for $\mathrm{H}$ ), suggesting the validity of the proposed structures. In addition, the in-situ variable temperature PXRD (VTPXRD) patterns of BrCOF-1 and BrCOF-2 at temperatures ranging from $30{ }^{\circ} \mathrm{C}$ to $350{ }^{\circ} \mathrm{C}$ demonstrate the high thermal stability of their crystalline structures, with negligible changes at $350{ }^{\circ} \mathrm{C}$ (Figure S10 and S11).
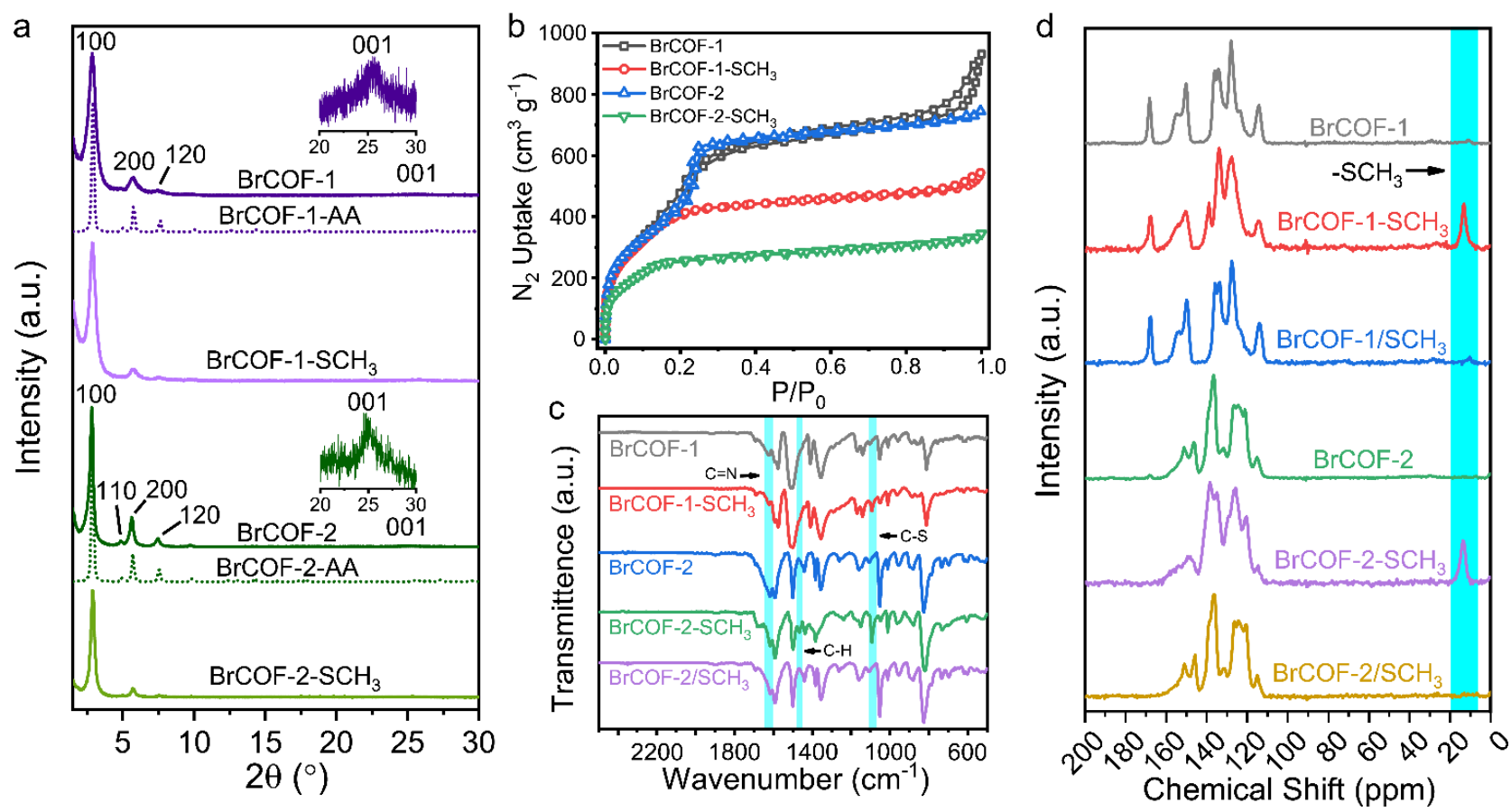

Figure 1. Characterizations of BrCOFs and the post-synthetically functionalized BrCOF-1-SCH 3 and $\mathrm{BrCOF}_{2}-\mathrm{SCH}_{3}$ via $\mathrm{Suzuki}_{-}$ Miyaura cross-coupling: (a) experimental and simulated PXRD patterns, (b) $\mathrm{N}_{2}$ sorption isotherms at 77K, (c) FT-IR spectra, and (d) Solid-state ${ }^{13} \mathrm{C}$ CP-MAS NMR spectra.

Brunauer-Emmett-Teller (BET) surface areas of BrCOF-1 and BrCOF-2 calculated from the nitrogen sorption analysis are as high as $1744 \mathrm{~m}^{2} \mathrm{~g}^{-1}$ and $1602 \mathrm{~m}^{2} \mathrm{~g}^{-1}$, respectively, and the pore volumes of these BrCOFs are up to $1.35 \mathrm{~cm}^{3} \mathrm{~g}^{-1}$ and $1.13 \mathrm{~cm}^{3} \mathrm{~g}^{-1}$ (Figure 1b). The pore size distributions of BrCOFs were generated by the non-local density functional theory (NLDFT), revealing that both BrCOFs adopt main peaks at around $2.5 \mathrm{~nm}$ (Figure S14 and S15), which are slightly smaller than those of simulated structures $(2.9 \mathrm{~nm})$. The minor discrepancy can be attributed to the layer slipping of 2D COFs. ${ }^{[5,35]}$ Still, the results illustrate that the pores of BrCOF-1 and BrCOF-2 are much larger than the size of the Pd catalyst tetrakis (triphenylphosphine) palladium $\left(\mathrm{Pd}\left(\mathrm{PPh}_{3}\right)_{4}, 1.3 \mathrm{~nm} \diamond 1.3\right.$ $\mathrm{nm} \diamond 1.1 \mathrm{~nm}$, Figure S16), making these BrCOFs suitable for the infiltration of the bulky Pd catalysts and the downstream modifications.

Featuring periodic structures, high specific surface areas, suitable pore sizes as well as abundant bromine atoms, the BrCOFs are considered to be ideal platforms for the post-synthetic modification via Suzuki-Miyaura crosscoupling. Given that sulfur atoms are absent from BrCOFs, which is convenient for evaluating the effects of the modification, the validity of this method was first demonstrated by modifying methylthio $\left(-\mathrm{SCH}_{3}\right)$ on the $\mathrm{BrCOFs}$. 
In general, a glass ampule was charged under argon (Ar) with BrCOF-1 or BrCOF-2 powder and 4(methylthio)phenylboronic acid in THF, following by the addition of aqueous $\mathrm{Cs}_{2} \mathrm{CO}_{3}$ solution and the catalyst $\mathrm{Pd}\left(\mathrm{PPh}_{3}\right)_{4}$. The Suzuki-Miyaura cross-coupling reaction was then carried out in $90{ }^{\circ} \mathrm{C}$ for $48 \mathrm{~h}$. Finally, the resulted wet product was activated under vacuum, yielding the solid product (designated as $\mathrm{BrCOF}-1-\mathrm{SCH}_{3}$ and $\mathrm{BrCOF}-2$ $\mathrm{SCH}_{3}$ ) before characterizations.

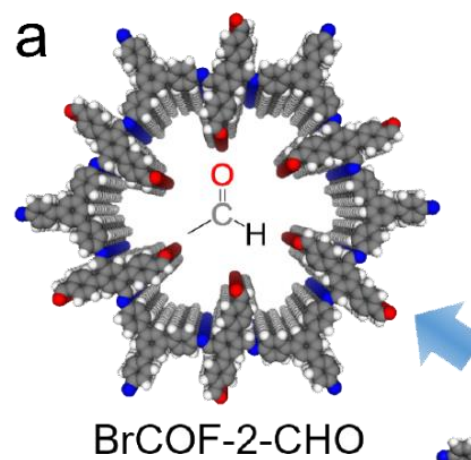

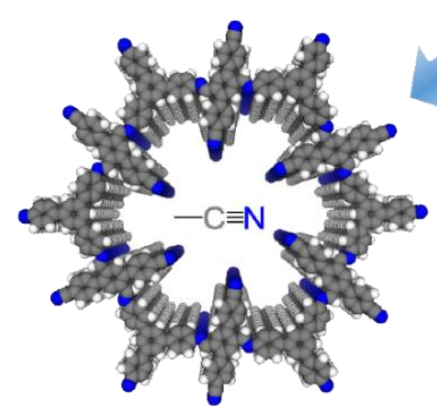

BrCOF-2-CN

b

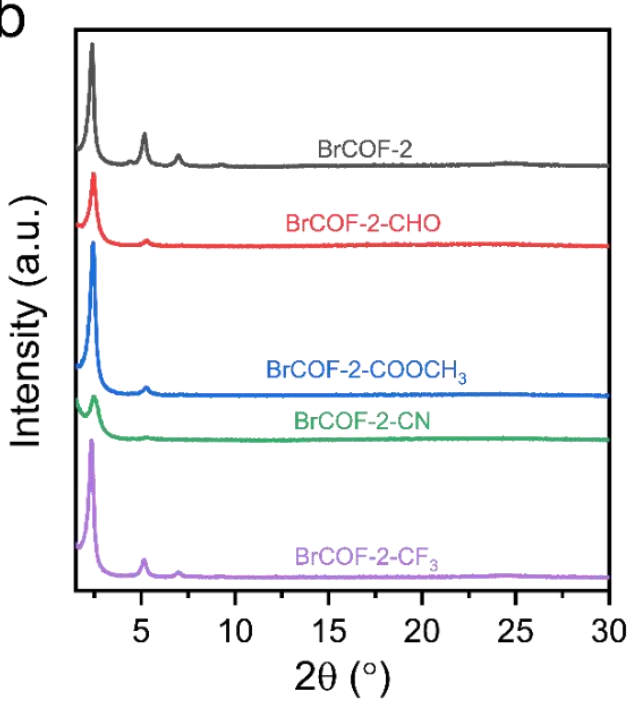

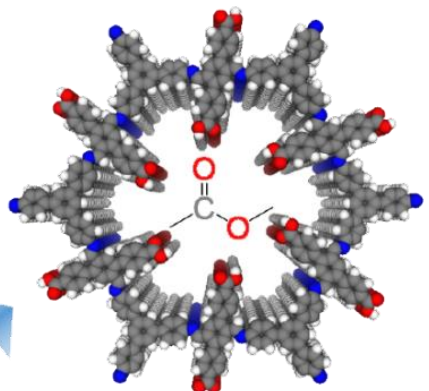

BrCOF-2- $\mathrm{COOCH}_{3}$

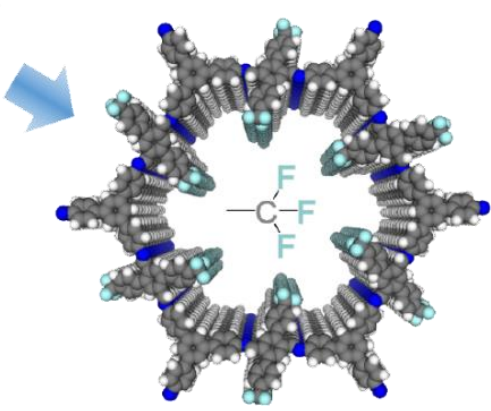

$\mathrm{BrCOF}-2-\mathrm{CF}_{3}$

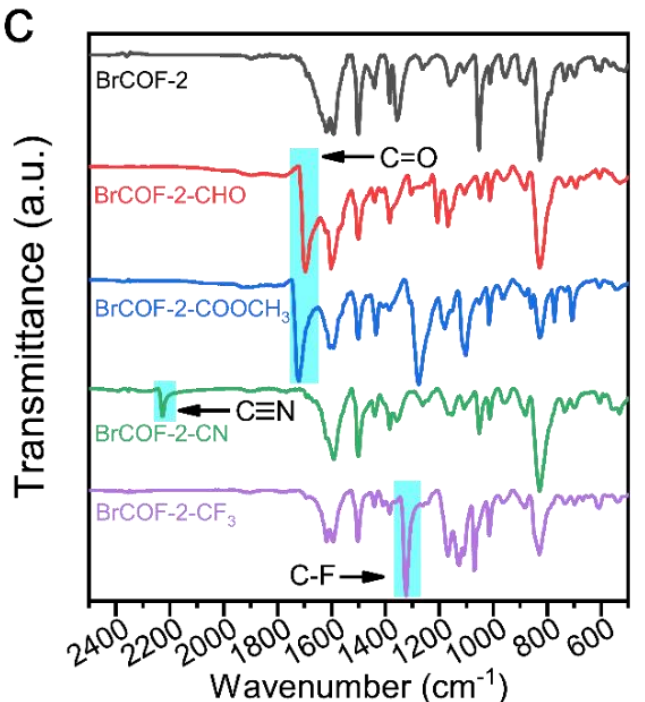

Figure 2. (a) Pore structures, (b) PXRD patterns and (c) FT-IR spectra of BrCOF-2, BrCOF-2-CHO, BrCOF-2-COOCH 3 , BrCOF-2$\mathrm{CN}$ and $\mathrm{BrCOF}-2-\mathrm{CF}_{3}$.

SEM images of the modified BrCOFs indicate no noticeable changes for the morphologies of post-synthetic modified COFs compared to those of bare BrCOFs (Figure S17). To further confirm the structural stability of postsynthetically functionalized BrCOFs, PXRD and $\mathrm{N}_{2}$ sorption analysis were conducted. As shown in Figure 1a, PXRD patterns of BrCOF-1-SCH 3 and $\mathrm{BrCOF}-2-\mathrm{SCH}_{3}$ show insignificant decline comparing those of their 
precursors, demonstrating the negligible influence of the Suzuki-Miyaura cross-coupling reaction on the crystalline structures of BrCOFs. $\mathrm{N}_{2}$ sorption analyses at $77 \mathrm{~K}$ reveal slightly decreased BET surface area of $1528 \mathrm{~m}^{2} \mathrm{~g}^{-1}$ and $1106 \mathrm{~m}^{2} \mathrm{~g}^{-1}$ for BrCOF-1-SCH 3 and BrCOF-2-SCH 3 after the reactions (Figure 1b), which can be attributed to the increased framework mass and the reduced pore volume as reported previously. ${ }^{[36]}$ Pore size distributions of BrCOF1-SCH 3 and BrCOF-2-SCH 3 calculated using the NLDFT model are also decreased from $2.5 \mathrm{~nm}$ to $1.3 \mathrm{~nm}$ (Figure S20 and S21). The chemical stabilities of these COFs were corroborated by intact FT-IR signals at around $1620 \mathrm{~cm}^{-}$ ${ }^{1}$ for the $\mathrm{C}=\mathrm{N}$ stretching vibration (Figure 1c).

In the FT-IR spectra of BrCOF-1-SCH 3 and BrCOF-2-SCH 3 , the C-S stretching vibrations at $1090 \mathrm{~cm}^{-1}$ and the $\mathrm{C}-\mathrm{H}$ deformation vibrations of methyl groups at $1465 \mathrm{~cm}^{-1}$ arise (Figure 1c), showing the appearance of methylthio groups. Successful modification is also manifested by ${ }^{13} \mathrm{C} \mathrm{CP} / \mathrm{MAS}$ NMR spectra (Figure 1d), in which significant peaks at $13 \mathrm{ppm}$ attributed to $-\mathrm{SCH}_{3}$ have emerged for both modified BrCOFs. From the results of elemental analysis, the sulfur contents of $\mathrm{BrCOF}-1-\mathrm{SCH}_{3}$ and $\mathrm{BrCOF}-2-\mathrm{SCH}_{3}$ are found to be $5.71 \mathrm{wt} \%$ and 6.97 $\mathrm{wt} \%$, respectively, corresponding to high $\mathrm{C}-\mathrm{Br}$ conversions of $47 \%$ and $59 \%$. As a control, BrCOFs powders were subjected to identical conditions in the absence of catalyst $\mathrm{Pd}\left(\mathrm{PPh}_{3}\right)_{4}$, resulting solids designed as $\mathrm{BrCOFs} / \mathrm{SCH}_{3}$. Both BrCOF-1/SCH 3 and BrCOF- $2 / \mathrm{SCH}_{3}$ show nearly no trace of sulfur elements $\left(0.22 \mathrm{wt} \%\right.$ for $\mathrm{BrCOF}-1 / \mathrm{SCH}_{3}$, $0.02 \mathrm{wt} \%$ for $\mathrm{BrCOF}-2 / \mathrm{SCH}_{3}$ ) and remain identical FT-IR and ${ }^{13} \mathrm{C} \mathrm{CP} / \mathrm{MAS}$ NMR spectra compared to those of BrCOFs (Figure 1c), corroborating the necessity of Pd-catalyst in this modification protocol for transferring C-Br bonds into C-C bonds. Additionally, the results demonstrate that high sulfur contents of $\mathrm{BrCOFs}_{-} \mathrm{SCH}_{3}$ are not originated from the adsorbed 4-(methylthio)phenylboronic acid molecules. Another control experiment was conducted under the same conditions except using the amorphous (Figure S22) and low-surface-area $\left(29 \mathrm{~m}^{2} \mathrm{~g}^{-1}\right.$, Figure S23) BrPOP-2, a chemical analog of BrCOF-2 confirmed by the FT-IR analysis (Figure S25), forming a product designated as $\mathrm{BrPOP}-2-\mathrm{SCH}_{3}$. The low sulfur content of $0.65 \mathrm{wt} \%$ testifies the low modification ratio of BrPOP-2-SCH . FT-IR spectrum of the modified product shows weak characteristic signals of the C-S bond and the methyl group (Figure S25), which can be attributed to the methylthio groups sparsely linked on the outer surface of particles. The results suggest that regular channels and large surface area of BrCOF-2 provide abundantly accessible reaction cites for the coupling reaction. On a basis of the above analyses, a conclusion is drawn out that BrCOFs can be modified with the methylthio group via Suzuki-Miyaura cross-coupling with well-preserved crystalline structures and porosities.

To examine the generality of the Suzuki-Miyaura cross-coupling approach, 4-(methylthio)phenylboronic acid was replaced with phenylboronic acids bearing formyl (-CHO), methoxycarbonyl (- $\left.\mathrm{COOCH}_{3}\right)$, cyano (-CN), or trifluoromethyl $\left(-\mathrm{CF}_{3}\right)$ functionalities to react with $\mathrm{BrCOF}-2$, aiming to produce corresponding functional COFs (designed as BrCOF-2-CHO, BrCOF-2- $\mathrm{COOCH}_{3}, \mathrm{BrCOF}-2-\mathrm{CN}$, and BrCOF-2- $\mathrm{CF}_{3}$, respectively, Figure 2a). As expected, the functionalization via Suzuki-Miyaura cross-coupling exerted only subtle effects on the morphology and crystallinity of BrCOF-2, which was testified by SEM images and PXRD analyses (Figure S26 and 2b). BET surface areas of BrCOF-2-CHO, BrCOF-2-COOCH 3 , BrCOF-2-CN, and BrCOF-2- $\mathrm{CF}_{3}$ determined by $\mathrm{N}_{2}$ sorption analyses remained as high as $683 \mathrm{~m}^{2} \mathrm{~g}^{-1}, 1029 \mathrm{~m}^{2} \mathrm{~g}^{-1}, 723 \mathrm{~m}^{2} \mathrm{~g}^{-1}$, and $1514 \mathrm{~m}^{2} \mathrm{~g}^{-1}$, respectively. In Figure 2c, FTIR spectra of these modified BrCOF-2s show remarkable characteristic peaks centering at $1697 \mathrm{~cm}^{-1}$ (for BrCOF2-CHO), $1720 \mathrm{~cm}^{-1}$ (for BrCOF-2-COOCH 3 ), $2231 \mathrm{~cm}^{-1}$ (for BrCOF-2-CN), and $1325 \mathrm{~cm}^{-1}$ (for BrCOF-2- $\mathrm{CF}_{3}$ ), respectively, confirming that $-\mathrm{CHO},-\mathrm{COOCH}_{3},-\mathrm{CN}$ and $-\mathrm{CF}_{3}$ have been successfully modified on the BrCOF-2. Elemental analyses revealed that the oxygen contents of BrCOF-2, BrCOF-2-CHO, and BrCOF-2-COOCH 3 were $3.48 \mathrm{wt} \%, 8.05 \mathrm{wt} \%$, and $9.12 \mathrm{wt} \%$, respectively, and the nitrogen content of BrCOF-2-CN was $7.03 \mathrm{wt} \%$. Besides, the fluorine content of BrCOF-2- $\mathrm{CF}_{3}$ was measured as $4.86 \pm 0.74 \mathrm{wt} \%$ using the EDX analysis. In accordance with the above values, the degrees of functionalization are estimated to be $78 \%, 49 \%, 28 \%$, and $23 \%$ for BrCOF-2-CHO, BrCOF-2-COOCH 3,3 BrCOF-2-CN, and BrCOF-2- $\mathrm{CF}_{3}$, respectively. These results unambiguously prove that Suzuki-Miyaura cross-coupling is a general and powerful tool to functionalize COFs, allowing for the facile introduction of desired functional groups. 


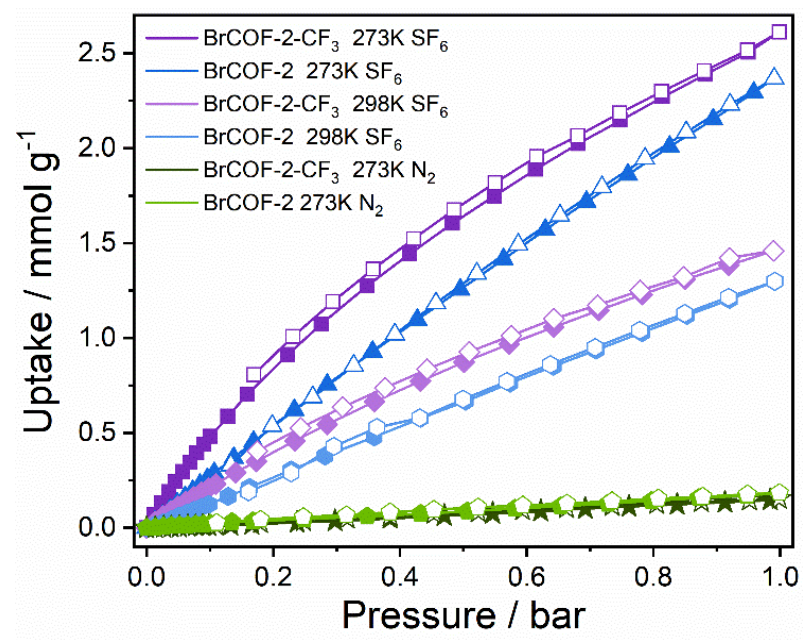

Figure 3. $\mathrm{SF}_{6}$ and $\mathrm{N}_{2}$ sorption isotherms at $273 \mathrm{~K}$ and $298 \mathrm{~K}$ of BrCOF-2 and BrCOF-2-CF 3.

Sulfur hexafluoride $\left(\mathrm{SF}_{6}\right)$ is a sort of valuable gas of widespread uses including electrical insulators, contrast agents, and plasma etchants. ${ }^{[37]}$ However, it is also the most potent greenhouse gas with a large global warming potential which is 23,900 times higher than $\mathrm{CO}_{2}$ and a long lifetime of 3200 years. ${ }^{[38,39]}$ To reduce the emission of $\mathrm{SF}_{6}$, pressure swing adsorption/desorption based on solid materials has been exploited as one of the efficient and energy-saving methods. ${ }^{[40]}$ Given the regular channels, suitable pore size, large remained porosity and abundant fluorine atoms which are fluorophilic, ${ }^{[41]} \mathrm{BrCOF}-2-\mathrm{CF}_{3}$ may act as a highly effective $\mathrm{SF}_{6}$ adsorbent for this method. Despite its smaller surface area, the modified BrCOF-2- $\mathrm{CF}_{3}$ shows significant $\mathrm{SF}_{6}$ uptakes of $2.61 \mathrm{mmol} \mathrm{g}^{-1}$ and $1.45 \mathrm{mmol} \mathrm{g}^{-1}$ at $273 \mathrm{~K}$ and 298K, 1 bar, which are higher than those of BrCOF-2 (Figure 3). It can also be found that BrCOF-2-CF 3 has higher isosteric heat of adsorption $\left(Q_{s t}\right)$ value than that of BrCOF-2 $\left(24.8 \mathrm{~kJ} \mathrm{~mol}^{-1}\right.$ vs. 20.6 $\mathrm{kJ} \mathrm{mol}^{-1}$, zero coverage, Figure S28), indicating the stronger interaction between the gas molecules and the pore walls of BrCOF-2-CF . The higher affinity of BrCOF-2-CF 3 can be ascribed to its narrow pore size of $1.3 \mathrm{~nm}$ upon modification, which is close to the ideal pore opening of $1.1 \mathrm{~nm}$ for $\mathrm{SF}_{6}$ adsorption. ${ }^{[2,43]}$ Additionally, the trifluoromethyl functionality renders $\mathrm{BrCOF}-2-\mathrm{CF}_{3}$ selective interaction between its pore walls and $\mathrm{SF}_{6}$ molecules. ${ }^{[44-46]}$

As displayed in Figure 3, the $\mathrm{SF}_{6}$ and $\mathrm{N}_{2}$ sorption isotherms of BrCOF-2 suggest its great potential for separating $\mathrm{SF}_{6}$ in $\mathrm{SF}_{6} / \mathrm{N}_{2}$ mixture. The $\mathrm{SF}_{6} / \mathrm{N}_{2}$ (10:90) selectivities at $273 \mathrm{~K}$ for BrCOF-2 and BrCOF-2-CF 3 were calculated using the ideally adsorbed solution theory (IAST) (Figure S29). At low-pressure region, BrCOF-2- $\mathrm{CF}_{3}$ exhibits high $\mathrm{SF}_{6} / \mathrm{N}_{2}$ selectivity of $\sim 53$ at 0.1 bar, which is 6.8 times larger than that of BrCOF-2. Although the selectivity for BrCOF-2- $\mathrm{CF}_{3}$ shows a minor decrease in the high-pressure region, it remains as high as $\sim 34$ at 1 bar, 2.5 times larger than that of BrCOF-2. The result further confirms the importance of the induction of trifluoromethyl to strengthen host-guest interactions

\section{Conclusion}

In summary, we have developed a brand-new method to functionalize COFs based on Suzuki-Miyaura crosscoupling. Thanks to the facile and effective Suzuki-Miyaura cross-coupling, a variety of functionalities were modified successfully on the pore walls of the readily available brominated COFs with high degrees of functionalization, while their crystallinity and porosity were well-retained. Aiming for $\mathrm{SF}_{6}$ adsorption and separation, BrCOF-2 was modified with $-\mathrm{CF}_{3}$ functionality to reduce the pore size and strengthen the binding with 
gas molecules, exhibiting significantly enhanced capability. Considering the ultra-large library of commercial reagents for Suzuki-Miyaura cross-coupling and multifarious methods to synthesize Suzuki monomers, this efficient protocol paves a new avenue towards the synthesis of functional COFs and provides almost unlimited possibilities for COF design targeting desired applications.

\section{Acknowledgments}

This work was supported by Shenzhen Science and Technology Innovation Committee (No. JCYJ20180307151441165) and the Fundamental Research Funds for the Central Universities (No. 020514380221).

Keywords: covalent organic frameworks $\bullet \mathrm{C}-\mathrm{C}$ coupling $\bullet$ brominated materials $\bullet$ post-synthetic modification $\bullet$ adsorption

\section{References}

[1] Q. Fang, Z. Zhuang, S. Gu, R. B. Kaspar, J. Zheng, J. Wang, S. Qiu, Y. Yan, A. P. Cote, A. I. Benin, et al., Science. 2005, 310 , $1166-1170$.

[2] S. Kandambeth, K. Dey, R. Banerjee, J. Am. Chem. Soc. 2019, 141, 1807-1822.

[3] X. Guan, F. Chen, Q. Fang, S. Qiu, Chem. Soc. Rev. 2020, 49, 1357-1384.

[4] W. Ji, L. Xiao, Y. Ling, C. Ching, M. Matsumoto, R. P. Bisbey, D. E. Helbling, W. R. Dichtel, J. Am. Chem. Soc. 2018, 140, 12677-12681.

[5] Q. Liao, C. Ke, X. Huang, G. Zhang, Q. Zhang, Z. Zhang, Y. Zhang, Y. Liu, F. Ning, K. Xi, J. Mater. Chem. A 2019, 7, 1895918970.

[6] Y. Ying, M. Tong, S. Ning, S. K. Ravi, S. B. Peh, S. C. Tan, S. J. Pennycook, D. Zhao, J. Am. Chem. Soc. 2020, 142, $4472-$ 4480 .

[7] H. Fan, M. Peng, I. Strauss, A. Mundstock, H. Meng, J. Caro, J. Am. Chem. Soc. 2020, 142, 6872-6877.

[8] E. Vitaku, C. N. Gannett, K. L. Carpenter, L. Shen, H. D. Abruña, W. R. Dichtel, J. Am. Chem. Soc. 2020, 142, 16-20.

[9] R. Shi, L. Liu, Y. Lu, C. Wang, Y. Li, L. Li, Z. Yan, J. Chen, Nat. Commun. 2020, 11, 178.

[10] S. Park, Z. Liao, B. Ibarlucea, H. Qi, H. Lin, D. Becker, J. Melidonie, T. Zhang, H. Sahabudeen, L. Baraban, et al., Angew. Chem. Int. Ed. 2020, 59, 8218-8224.

[11] Y. Xiong, Q. Liao, Z. Huang, X. Huang, C. Ke, H. Zhu, C. Dong, H. Wang, K. Xi, P. Zhan, et al., Adv. Mater. 2020, 32, 1907242.

[12] M. Lu, J. Liu, Q. Li, M. Zhang, M. Liu, J. Wang, D.-Q. Yuan, Y.-Q. Lan, Angew. Chem. Int. Ed. 2019, 58, $12392-12397$.

[13] Q. Liao, W. Xu, X. Huang, C. Ke, Q. Zhang, K. Xi, J. Xie, Sci. China Chem. 2020, 63, 707-714.

[14] G. Zhang, X. Li, Q. Liao, Y. Liu, K. Xi, W. Huang, X. Jia, Nat. Commun. 2018, 9, 2785.

[15] S. Liu, J. Yang, R. Guo, L. Deng, A. Dong, J. Zhang, Macromol. Rapid Commun. 2020, 41, 1900570.

[16] P. J. Waller, S. J. Lyle, T. M. Osborn Popp, C. S. Diercks, J. A. Reimer, O. M. Yaghi, J. Am. Chem. Soc. 2016, 138, 1551915522.

[17] J. L. Segura, S. Royuela, M. M. Ramos, Chem. Soc. Rev. 2019, 48, 3903-3945.

[18] Y. Li, Q. Chen, T. Xu, Z. Xie, J. Liu, X. Yu, S. Ma, T. Qin, L. Chen, J. Am. Chem. Soc. 2019, 141, 13822-13828.

[19] Y. Yusran, X. Guan, H. Li, Q. Fang, S. Qiu, Natl. Sci. Rev. 2020, 7, 170-190.

[20] H. Ding, A. Mal, C. Wang, Mater. Chem. Front. 2020, 4, 113-127.

[21] A. Nagai, Z. Guo, X. Feng, S. Jin, X. Chen, X. Ding, D. Jiang, Nat. Commun. 2011, 2, 536.

[22] N. Huang, R. Krishna, D. Jiang, J. Am. Chem. Soc. 2015, 137, 7079-7082.

[23] D. N. Bunck, W. R. Dichtel, Chem. Commun. 2013, 49, 2457-2459.

[24] Q. Sun, B. Aguila, J. Perman, L. D. Earl, C. W. Abney, Y. Cheng, H. Wei, N. Nguyen, L. Wojtas, S. Ma, J. Am. Chem. Soc. 2017, 139, 2786-2793. 
[25] Y. Fu, Z. Wang, X. Fu, J. Yan, C. Liu, C. Pan, G. Yu, J. Mater. Chem. A 2017, 5, 21266-21274.

[26] D. H. Howe, R. M. Mcdaniel, A. J. D. Magenau, Macromolecules 2017, 50, 8010-8018.

[27] N. Miyaura, K. Yamada, A. Suzuki, Tetrahedron Lett. 1979, 20, 3437-3440.

[28] N. Miyaura, A. Suzuki, J. Chem. Soc., Chem. Commun. 1979, 866-867.

[29] N. Miyaura, A. Suzuki, Chem. Rev. 1995, 95, 2457-2483.

[30] K. C. Nicolaou, P. G. Bulger, D. Sarlah, Angew. Chem. Int. Ed. 2005, 44, 4442-4489.

[31] C. C. C. Johansson Seechurn, M. O. Kitching, T. J. Colacot, V. Snieckus, Angew. Chem. Int. Ed. 2012, 51, 5062-5085.

[32] K. Nakabayashi, Y. Abiko, H. Mori, Macromolecules 2013, 46, 5998-6012.

[33] T. S. Jo, S. H. Kim, J. Shin, C. Bae, J. Am. Chem. Soc. 2009, 131, 1656-1657.

[34] L. Lercher, J. F. Mcgouran, B. M. Kessler, C. J. Schofield, B. G. Davis, Angew. Chem. Int. Ed. 2013, 52, 10553-10558.

[35] Q. Fang, Z. Zhuang, S. Gu, R. B. Kaspar, J. Zheng, J. Wang, S. Qiu, Y. Yan, Nat. Commun. 2014, 5, 4503.

[36] X. Li, C. Zhang, S. Cai, X. Lei, V. Altoe, F. Hong, J. J. Urban, J. Ciston, E. M. Chan, Y. Liu, Nat. Commun. $2018,9,2998$.

[37] T. Hasell, M. Miklitz, A. Stephenson, M. A. Little, S. Y. Chong, R. Clowes, L. Chen, D. Holden, G. A. Tribello, K. E. Jelfs, et al., J. Am. Chem. Soc. 2016, 138, 1653-1659.

[38] A. A. R. Ravishankara, S. Solomon, A. A. Turnipseed, R. F. Warren, Science 1993, 259, 194-199.

[39] S. Solomon, D. Qin, M. Manning, M. Marquis, Climate Change 2007-the Physical Science Basis: Working Group I Contribution to the Fourth Assessment Report of the IPCC, Cambridge University Press, 2007.

[40] R. T. Yang, Adsorbents:Fundamentals and Applications, John Wiley \& Sons, 2003.

[41] T. Chen, I. Popov, W. Kaveevivitchai, Y. Chuang, Y. Chen, A. J. Jacobson, S. Ognjen, Angew. Chem. Int. Ed. 2015, 54, 1390213906.

[42] S. Builes, T. Roussel, L. F. Vega, Aiche J. 2011, 57, 962-974.

[43] M. B. Kim, S. J. Lee, C. Y. Lee, Y. S. Bae, Microporous Mesoporous Mater. 2014, 190, 356-361.

[44] T. H. Chen, I. Popov, W. Kaveevivitchai, Y. C. Chuang, Y. S. Chen, A. J. Jacobson, O. S. Miljanic, Angew. Chem. Int. Ed. 2015, 54, 13902-13906.

[45] Y. Koda, T. Terashima, M. Sawamoto, J. Am. Chem. Soc. 2014, 136, 15742-15748.

[46] T. H. Chen, I. Popov, W. Kaveevivitchai, Y. C. Chuang, Y. S. Chen, O. Daugulis, A. J. Jacobson, O. Miljanić, Nat. Commun. 2014, $5,5131$.

\section{Entry for the Table of Contents}
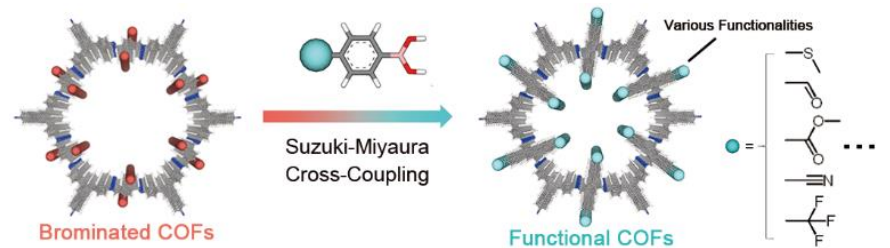

A versatile and efficient post-synthetic modification (PSM) method for functionalizing COFs via Suzuki-Miyaura cross-coupling has been developed in this article. By this means, various functionalities have been successfully installed on the pore walls of brominated COFs, producing a batch of novel functional COFs. The trifluoromethyl functionalization endows the $\mathrm{COF}$ with significantly enhanced properties for $\mathrm{SF}_{6}$ adsorption and separation. 